\title{
Variantes emergentes do SARS-CoV-2 e suas implicações na saúde coletiva
}

\author{
André Ricardo Ribas Freitas*1 \\ Marta Giovanetti² \\ Luiz Carlos Junior Alcantara2,3
}

\begin{abstract}
${ }^{1}$ Disciplina de Epidemiologia, Bioestatística e Metodologia Científica, Faculdade São Leopoldo Mandic, Campinas-SP
${ }^{2}$ Laboratorio de Flavivirus, Fundacao Oswaldo Cruz, Rio de Janeiro, Brazil

${ }^{3}$ Laboratorio de Genetica Celular e Molecular, ICB, Universidade Federal de Minas Gerais, Belo Horizonte, Brazil

*Corresponding author: andre.freitas@slmandic.edu.br
\end{abstract}

Todos os vírus, incluindo o SARS-CoV-2 (do inglês: Severe Acute Respiratory Syndrome CoronaVirus 2) sofrem mutação. Essas mudanças genéticas acontecem à medida que o vírus faz novas cópias de si mesmo para se espalhar e prosperar. A maioria das mutações é irrelevante e algumas podem até ser prejudiciais à perpetuação do vírus (pressão seletiva negativa), mas outras podem facilitar sua propagação (pressão seletiva positiva) ou torna-lo mais patogênico para o hospedeiro (os seres humanos).

Entre as mutações do SARS-CoV-2 mais relevantes do ponto de vista clínico e epidemiológico estão as que afetam a proteína $S$ (do inglês, spike, espicula viral), que desempenha um papel importante durante o processo infeccioso facilitando a entrada do coronavírus nas células humanas. O processo de infecção acontece quando o vírus entra na célula após a ligação entre o domínio RBD (do inglês receptor-binding domain) da proteína $S$ e o receptor da célula alvo, que é a enzima conversora de angiotensina 2 (ACE-2)[1].

Grande parte da proteção contra a COVID-19 (do inglês: COronaVIrus Disease 2019) é mediada pela resposta imune dirigida contra esta mesma proteína. Anticorpos neutralizantes do vírus ligam-se à proteína $S$, geralmente na região do RBD impedindo que o vírus se fixe ao receptor ACE-2 das células humanas. Grande parte, se não a totalidade, das vacinas atualmente em uso ou em estudo se baseiam na indução da produção de anticorpos direcionados a estes sítios[1].

Mutações na proteína S que aumentam a quantidade de vírus eliminado por uma pessoa infectada ou que aumentam sua afinidade do RBD pelo receptor ACE2 podem estar associadas ao aumento da transmissão do vírus. Um exemplo é a mutação D614G que está associada a cargas virais mais altas e a uma maior transmissibilidade. Linhagens com esta mutação passaram a ser predominantes em todo o mundo a partir de meados de 2020, substituindo as linhagens originais[2]. Outras mutações podem alterar a proteína $\mathrm{S}$ e prejudicar a ligação dos anticorpos neutralizantes e, consequentemente, aumentar o risco de reinfecções ou diminuir a eficácia das vacinas. Outro aspecto a ser considerado das mutações é a perda de sensibilidade aos testes de diagnóstico, que traz preocupação quanto à necessidade do monitoramento contínuo das novas variantes para permitir a continua atualização dos métodos de diagnostico utilizados no screening molecular ou mesmo sorológico.

Nas últimas semanas tem surgido no meio científico uma grande preocupação em relação a três variantes do vírus SARS-CoV-2, conhecidas na nomenclatura dinâmica, recentemente proposta por Rambaut et al., [3] como: i) B.1.1.7 (ou VUI - 202012/01), ii) B.1.351 (ou 501Y.V2) e iii) P.1, inicialmente identificadas em pacientes infectados respectivamente no Reino Unido, na África do Sul e em Manaus, no Brasil [4-6].

Essas variantes são consideradas preocupantes devido a presença de um conjunto de mutações que levaram ao aumento da transmissibilidade e à deterioração das situações epidemiológicas nas áreas onde recentemente 
se estabeleceram. Apesar de terem origem distintas, elas compartilham uma constelação de mutações, o que reforça a possibilidade de que estas mutações ofereçam vantagens competitivas relevantes.

\section{Variantes preocupantes (VOC, variant of concern)}

Em 14 de dezembro de 2020, as autoridades do Reino Unido notificaram a OMS uma variante denominada pelo Reino Unido como SARS-CoV-2 VOC 202012/01 ou B.1.1.7. Essa linhagem carrega 14 mutações definidoras, incluindo sete na proteína S (Tabela-1). Entre estas 7 está a mutação N501Y, que está associada a maior afinidade do vírus pelo receptor $\mathrm{ACE}-2$ o que pode explicar a sua rápida expansão [4]. O nome da mutação se refere a natureza da sua mudança: o aminoácido na posição 501 na proteína $\mathrm{S}$ mudou de $\mathrm{N}$ (asparagina) para Y (tirosina). Além disso, essa linhagem carrega algumas deleções entre elas as que excluem os aminoácidos 69 e o 70 da proteína S. Experimentos recentes mostraram que essa "eliminação" de aminoácidos permite que o novo coronavirus infecte as células com mais sucesso. Até o momento essa linhagem já foi encontrada em 70 países, dos quais 29 com transmissão local. Ela foi responsabilizada pelo agravamento da situação epidemiológica no Reino Unido, Portugal e outros países da Europa entre dezembro de 2020 e janeiro de 2021. Apesar dos primeiros relatos não encontrarem evidências de impacto na gravidade da doença, uma atualização publicada em janeiro de 2021 do NERVTAG (New and Emerging Respiratory Virus Threats Advisory Group) da agência britânica Public Health England encontrou, entre outros, um estudo de coorte que encontrou um risco relativo de morte de 1,65 (IC de 95\% 1,21-2,25) entre indivíduos infectados com a linhagem B.1.1.7 comparado com casos não-B.1.1.7[7]. Outro achado preocupante em relação à esta linhagem foi o aumento na proporção de casos entre crianças e adultos jovens [8].

Em 18 de dezembro de 2020, as autoridades nacionais da África do Sul anunciaram a detecção de uma nova variante do SARS-CoV-2 denominada B.1.351 (ou 501Y.V2), devido à presença da mutação N501Y. Embora a variante B.1.1.7 também tenha a mutação N501Y, análises filogenéticas demonstraram que a variante B.1.351, detectada na África do Sul, tem origem diferente. Os dados genômicos demonstraram que a variante 501Y. V2 substituiu rapidamente outras linhagens que circulam na África do Sul se tornando a dominante na região [5]. A linhagem B.1.351 apresenta múltiplas mudanças na proteína $S$ das quais se destacam três que estão localizadas no domínio RDB (K417N, E484K e N501Y) (Tabela-1). A mutação N501Y variante está associada ao aumento da infecciosidade, uma vez que ela parece aumentar a ligação entre a proteína $\mathrm{S}$ do vírus e o receptor do hospedeiro. A mutação E484K, altera a região da espícula onde ocorre o acoplamento de imunoglobulinas neutralizantes permitindo o escape viral (quando o vírus não é neutralizado pelo anticorpo) e aumentando o risco de reinfecção [9]. Essa linhagem foi identificada pela primeira vez na Baía Nelson Mandela, ao longo da costa leste da África do Sul e espalhouse rapidamente para os outros distritos do Cabo Oriental e para as província do Cabo Ocidental e KwaZulu Natal $(K Z N)$, tornando-se a linhagem dominante nas províncias do Cabo Oriental e do Cabo Ocidental em semanas [5]. Já foi encontrada até o momento em 31 países, dos quais 13 com transmissão local, e está associada à segunda onda de COVID-19 iniciada em dezembro de 2021 na África do Sul (https://cov-lineages.org/global report.html, consultado em 6/fev/2021).

Em 9 de janeiro de 2021, o Japão notificou à OMS sobre uma nova variante de SARS-CoV-2, a P1 (inicialmente relatada como B.1.1.248), detectada em quatro viajantes provenientes do Brasil. Esta variante não está geneticamente relacionada às variantes SARS-CoV-2 B.1.1.7 e B.1.351, tendo sido identificada em dezembro de 2020 em Manaus, capital do estado do Amazonas, no Brasil. Essa variante possui 12 mutações na proteína $S$, incluindo três mutações de interesse em comum com B.1.351, ou seja, K417N / T, E484K e N501Y, que podem afetar a transmissibilidade e a resposta imune do hospedeiro (Tabela 1).

O fato dessas linhagens de terem origens distintas, mas possuírem as mesmas mutações, sugere um processo denominado convergência evolutiva, que é o nome dado quando características semelhantes são selecionadas em diferentes locais por representarem vantagens claras como maior transmissibilidade, sucesso na replicação ou mesmo escape imunológico.

Esta nova linhagem de SARS-CoV-2 esteve ausente nas amostras coletadas entre março a novembro em Manaus, mas foi identificada em $42 \%$ das amostras de dezembro de 2020 e em $91 \%$ das amostras coletadas na mesma cidade em janeiro de 2021 (Figura-1), sugerindo um forte e recente aumento na frequência desta linhagem 
associada à segunda e maior onda de COVID-19 na cidade [6]. A soroprevalência em Manaus observada em junho de 2020 era de 52,5\% (IC 95\%= 47,6-57,5), logo após a primeira onda epidêmica indicando que esta nova linhagem foi, também, submetida à uma forte pressão seletiva [10].

Também merece ser citada, a linhagem brasileira P.2, portadora da mutação E484K, identificada pela primeira vez em outubro de 2020, já era a mais prevalente entre as linhagens sequenciadas de pacientes que desenvolveram sintomas em novembro no estado do Rio de Janeiro, também no Brasil. [11]. Analisando os dados da Rede Genômica Fiocruz, que reúne pesquisadores de diversos institutos da Fundação Oswaldo Cruz, verificamos que desde o início da emergência das linhagens P.1 e P.2 em outubro de 2020, em apenas 4 meses estas linhagens correspondiam juntas por $75 \%$ de todas as linhagens sequenciadas em todo o Brasil (Figura 1). Na cidade de Manaus estas duas linhagens juntas corresponderam à $97,8 \%$ das amostras de vírus sequenciados em janeiro de 2021 (Figura 1).

Além das variantes já mencionadas, o Brasil, Europa, os Estados Unidos da América, o Japão e outros países já notificaram a detecção de muitas outras novas variantes, cuja abrangência e importância para a saúde pública exigem mais investigação epidemiológica e laboratorial.

\section{Reinfecções}

O fato de novas linhagens causarem epidemias em locais já afetados por epidemias severas anteriores levanta algumas preocupações tais como o de aumento na transmissibilidade e da possibilidade do escape antigênico[12]. Apontando neste sentido, no Brasil vários casos de reinfecção pelas novas linhagens também têm sido descritos [13-15] .

A descoberta de casos de reinfeccao serve de alerta e reforça a necessidade de manutenção das medidas de controle da pandemia, com distanciamento social e a necessidade de acelerar o processo de vacinação, para reduzir a possibilidade de circulação desta e de possíveis futuras linhagens que, ao acumular mutações, podem vir a se tornar mais infectantes, inclusive para indivíduos que já tiveram a doença [12]. Nesse sentido, já foi recentemente demonstrado in vitro que a linhagem B.1.351 tem maior resistência à neutralização por anticorpos de pacientes que tiveram infecção natural [16].

\section{Vacinas}

Estudos sobre a proteção entre pessoas que receberam vacinas contra COVID-19 ainda são poucos, mas já demonstram que a resposta a estas linhagens é diferente das respostas às linhagens originais. Pesquisadores estimaram que a vacina Novavax foi 95,6\% eficaz contra o vírus que circulava originalmente no Reino Unido e 85,6\% contra a linhagem B.1.1.7 [17]. De fato, mutação N501Y, associada a esta linhagem, levantava menos preocupações sobre a capacidade do escape imunológico. Os estudos realizados com esta vacina na África do Sul demonstram uma eficácia global de 49,4\% contra a linhagem B.1.351, excluindo a população HIVpositiva desse grupo da análise, a vacina foi $60 \%$ eficaz [17]. A atividade de plasma de indivíduos 8 semanas após a segunda dose das vacinas de mRNA Moderna (mRNA1273) ou Pfizer-BioNTech (BNT162b2) contra as variantes do SARS-CoV-2 que carregam as mutações E484K ou N501Y ou a combinação K417N: E484K: N501Y foi reduzida por uma margem pequena, mas significativa. [18].

As taxas de proteção das vacinas contra as novas linhagens emergentes ainda não foram total e definitivamente testadas em estudos de fase 3, nem sabemos como será o comportamento da proteção induzida por qualquer vacina ao longo do tempo (Tabela 2). Mas fica o alerta sobre a possibilidade de que a proteção da infecção natural (e das vacinas, por extensão) possam não ser tão duradouras e que a proteção contra as novas linhagens não seja tão eficiente.

\section{Vigilância virológica, rastreamento e quarentena de contatos}

O início da vacinação em todo o mundo tem sido bastante comemorado e é realmente um fato bastante positivo, no entanto, é necessário cautela com o relaxamento das medidas de controle desta doença e principalmente com a comunicação social de risco, uma vez que a população precisa se conscientizar da necessidade de manutenção dos cuidados de afastamento social e cuidados individuais para diminuição do risco de transmissão do SARS-CoV-2. 
Considerando a ocorrência de grandes epidemias onde já haviam ocorrido graves ondas epidêmicas, o aumento no risco de reinfecções e até a possibilidade de diminuição na eficácia das vacinas, é fundamental reforçar a vigilância virológica e genômica para identificar precocemente e acompanhar a difusão de novas linhagens, sejam elas brasileiras, sejam elas recém introduzidas [19].

Uma vigilância específica buscando investigar adequadamente e sequenciar os vírus causadores de infecções em vacinados ou reinfecções é mandatório para que novas linhagens e/ou escape imunológico possam ser identificados precocemente e medidas de contenção sejam tomadas.

No Brasil o controle da COVID-19 tem se baseado fundamentalmente nas medidas coletivas de afastamento social, o rastreamento e quarentena de contatos não tem sido aplicado sistematicamente pelos órgão de saúde pública brasileiro em nenhum dos três níveis de governo sejam eles federal, estadual e municipal. Considerando que mais quase $50 \%$ da transmissão ocorre antes do início dos sintomas, o rastreamento e quarentena de contatos se torna fundamental para a contenção destas novas linhagens e é estratégico para garantir o controle da doença, ou pelo menos para a diminuição da velocidade de transmissão enquanto mais estudos podem ser feitos para aumentar os conhecimentos e capacidade de enfrentamento a estas novas linhagens.

Por tudo o que foi apresentado acreditamos que o desafio imposto pelo SARS-CoV-2 não termina com a introdução das vacinas, muito ainda deve ser feito nos próximos meses e anos em termos de vigilância epidemiológica, virológica e estudos nas áreas de epidemiologia, clínica, imunologia, virologia e outras áreas do conhecimento para garantir segurança à população mundial em relação à COVID-19.

\section{Bibliografia}

[[1] Dai L, Gao GF. Viral targets for vaccines against COVID-19. Nature Reviews Immunology 2020; 21: 73-82.

[2] Volz E, Hill V, McCrone JT, et al. Evaluating the Effects of SARS-CoV-2 Spike Mutation D614G on Transmissibility and Pathogenicity. Cell 2021; 184: 64-75.e11.

[3] Rambaut A, Holmes EC, O'Toole Á, et al. A dynamic nomenclature proposal for SARS-CoV-2 lineages to assist genomic epidemiology. Nat Microbiol 2020; 5: 1403-1407.

[4] Rambaut A, Loman N, Pybus O, etal. Preliminary genomic characterisation of an emergent SARS-CoV-2 lineage in the UK defined by a novel set of spike mutations - SARSCoV-2 coronavirus / nCoV-2019 Genomic Epidemiology - Virological. virological.org, https://virological.org/t/ preliminary-genomic-characterisation-of-an-emergentsars-cov-2-lineage-in-the-uk-defined-by-a-novel-set-ofspike-mutations/563 (2020, accessed 7 February 2021).

[5] Tegally $H$, Wilkinson $E$, Giovanetti $M$, et al Emergence and rapid spread of a new severe acute respiratory syndrome-related coronavirus 2 (SARSCoV-2) lineage with multiple spike mutations in South Africa. MedRxiv 2020; 10: 2020.12.21.20248640.

[6] Faria NR, Claro IM, Candido D, et al. Genomic characterisation of an emergent SARS-CoV-2 lineage in Manaus: preliminary findings - SARS-CoV-2 coronavirus / nCoV-2019 Genomic Epidemiology-Virological. virological. org, https://virological.org/t/genomic-characterisation-ofan-emergent-sars-cov-2-lineage-in-manaus-preliminaryfindings/586 (2021, accessed 7 February 2021).

[7] Horby P, Huntley C, Davies $N$, et al NERVTAG paper on COVID-19 variant of concern B.1.1.7. London, 1 January 2021.

[8] LeeR.B.1.1.7:WhatWeKnowAbouttheNovelSARSCoV-2 Variant. asm.org, https://asm.org/Articles/2021/ January/B-1-1-7-What-We-Know-About-the-NovelSARS-CoV-2-Va (2021, accessed 7 February 2021).

[9] Nelson G, Buzko O, Patricia S, et al. Molecular dynamic simulation reveals E484K mutation enhances spike RBD-ACE2 affinity and the 1 combination of E484K, K417N and N501Y mutations (501Y.V2 variant) induces conformational 2 change greater than N501Y mutant alone, potentially resulting in an escape mutant 3 4. bioRxiv 2021; 2021.01.13.426558.

[10] BussL,PreteC,Abrahim C, etal. Three-quartersattack rate of SARS-CoV-2 in the Brazilian Amazon during a largely unmitigated epidemic. Science (80- ) 2020; 371: 288-292. 
[11] Voloch CM, FR daS, Almeida LGP de, etal. Genomic characterization of a novel SARS-CoV-2 lineage from Rio de Janeiro, Brazil. MedRxiv 2020; 2020.12.23.20248598.

[12] Sabino EC, Buss LF, Carvalho MPS, et al. Resurgence of COVID-19 in Manaus, Brazil, despite high seroprevalence. Lancet 2021; 397: 452-455.

[13] Resende PC, Bezerra JF, Vasconcelos RHT de, et al. Spike E484K mutation in the first SARS-CoV-2 reinfection case confirmed in Brazil, 2020 - SARS-CoV-2 coronavirus / nCoV-2019 Genomic Epidemiology-Virological. virological. org, https://virological.org/t/spike-e484k-mutationin-the-first-sars-cov-2-reinfection-case-confirmed-inbrazil-2020/584 (2021, accessed 7 February 2021).

[14] Kymie C, Nonaka V, Franco MM, et al. Genomic evidence of a SARS-CoV-2 reinfection case with E484K spike mutation in Brazil. Preprints, https:// www.gisaid.org/ (2021, accessed 7 February 2021).

[15] Naveca F, Costa $C$ da, Nascimento $V$, et al. SARS-CoV-2 reinfection by the new Variant of Concern (VOC) P.1 in Amazonas, Brazil - SARS-CoV-2 coronavirus I nCoV-2019 Genomic Epidemiology - Virological. virological.org, https://virological.org/t/sars-cov-2reinfection-by-the-new-variant-of-concern-voc-p-1-inamazonas-brazil/596 (2021, accessed 7 February 2021).

[16] Wibmer CK, Ayres F, Hermanus T, et al. SARS-CoV-2 501Y.V2 escapes neutralization by South African COVID-19 donor plasma. BioRxiv 2021; 2021.01.18.427166.

[17] Novavax Inc. Novavax COVID-19 Vaccine Demonstrates $89.3 \%$ Efficacy in UK Phase 3, https://ir.novavax.com/node/15506/ pdf (2021, accessed 7 February 2021).

[18] Wang $Z$, Schmidt $F$, Weisblum $Y$, et al. mRNA vaccine-elicited antibodies to SARS-CoV-2 and circulating variants 234 . bioRxiv 2021; 2021.01.15.426911.

[19] Claro IM, da Silva Sales FC, Ramundo MS, et al. Local Transmission of SARS-CoV-2 Lineage B.1.1.7, Brazil, December 2020. Emerg Infect Dis; 27. Epub ahead of print 26 January 2021. DOI: 10.3201/eid2703.210038. 
Table 1. Variantes emergentes que causam preocupação na atualidade. (Fonte: https://cov-lineages.org/)

\begin{tabular}{|c|c|c|}
\hline P.1 & $\begin{array}{c}\text { aa:orf1ab:S1188L } \\
\text { aa:orf1ab:K1795Q } \\
\text { del:11288:9 } \\
\text { aa:S:L18F } \\
\text { aa:S:T20N } \\
\text { aa:S:P26S } \\
\text { aa:S:D138Y } \\
\text { aa:S:R190S } \\
\text { aa:S:K417T } \\
\text { aa:S:E484K } \\
\text { aa:S:N501Y } \\
\text { aa:S:H655Y } \\
\text { aa:S:T1027I } \\
\text { aa:orf3a:G174C } \\
\text { aa:orf8:E92K } \\
\text { aa:N:P80R }\end{array}$ & $\begin{array}{l}\text { Brazil 66, Japan 4, USA 3, Italy 3, France 3, Netherlands 2, South_Korea 1, } \\
\text { Faroe_Islands 1, Peru } 1\end{array}$ \\
\hline B.1.1.7 & $\begin{array}{c}\text { aa:orf1ab:T1001। } \\
\text { aa:orf1ab:A1708D } \\
\text { aa:orf1ab:I2230T } \\
\text { del:11288:9 } \\
\text { del:21765:6 } \\
\text { del:21991:3 } \\
\text { aa:S:N501Y } \\
\text { aa:S:A570D } \\
\text { aa:S:P681H } \\
\text { aa:S:T716I } \\
\text { aa:S:S982A } \\
\text { aa:S:D1118H } \\
\text { aa:Orf8:Q27* } \\
\text { aa:Orf8:R52I } \\
\text { aa:Orf8:Y73C } \\
\text { aa:N:D3L } \\
\text { aa:N:S235F }\end{array}$ & $\begin{array}{l}\text { UK 46042, Denmark 1076, France 472, Netherlands 466, USA 404, Belgium } \\
\text { 402, Spain 350, Ireland 276, Switzerland 211, Portugal 201, Italy 169, Austria } \\
\text { 140, Turkey 116, Israel 78, Germany 77, Australia 71, Sweden 66, Finland 42, } \\
\text { Singapore 40, Jordan 40, Slovakia 37, Norway 33, Luxembourg 32, New_Zea- } \\
\text { land 26, India 22, United_Arab_Emirates 21, Iceland 20, Brazil 19, Mayotte } \\
\text { 18, Romania 13, Nigeria 13, Czech_Republic 13, South_Korea 13, Poland 9, } \\
\text { Ecuador 6, North_Macedonia 5, Hungary 5, Greece 4, Jamaica 4, Latvia 4, } \\
\text { St._Lucia 3, Thailand 3, Canada 3, Hong_Kong 3, Bangladesh 3, Gambia 3, } \\
\text { Malaysia 2, Pakistan 2, Mexico 1, Slovenia } 1\end{array}$ \\
\hline B.1.351 & $\begin{array}{c}\text { aa:E:P71L } \\
\text { aa:N:T205I } \\
\text { aa:orf1a:K1655N } \\
\text { aa:S:D80A } \\
\text { aa:S:D215G } \\
\text { aa:S:K417N } \\
\text { aa:S:A701V } \\
\text { aa:S:N501Y } \\
\text { aa:S:E484K }\end{array}$ & $\begin{array}{l}\text { South_Africa 642, UK 114, Belgium 37, Netherlands 31, France 29, Mayotte } \\
\text { 21, Mozambique 19, Botswana 14, Switzerland 11, Germany 10, Australia 10, } \\
\text { Ireland 9, New_Zealand 7, Denmark 5, USA 5, United_Arab_Emirates 5, Fin- } \\
\text { land 2, Turkey 2, Spain 2, Kenya 2, Luxembourg 2, Portugal 1, South_Korea 1, } \\
\text { Sweden 1, Gaborone 1, Norway 1, Panama 1, Austria 1, Bangladesh } 1\end{array}$ \\
\hline
\end{tabular}




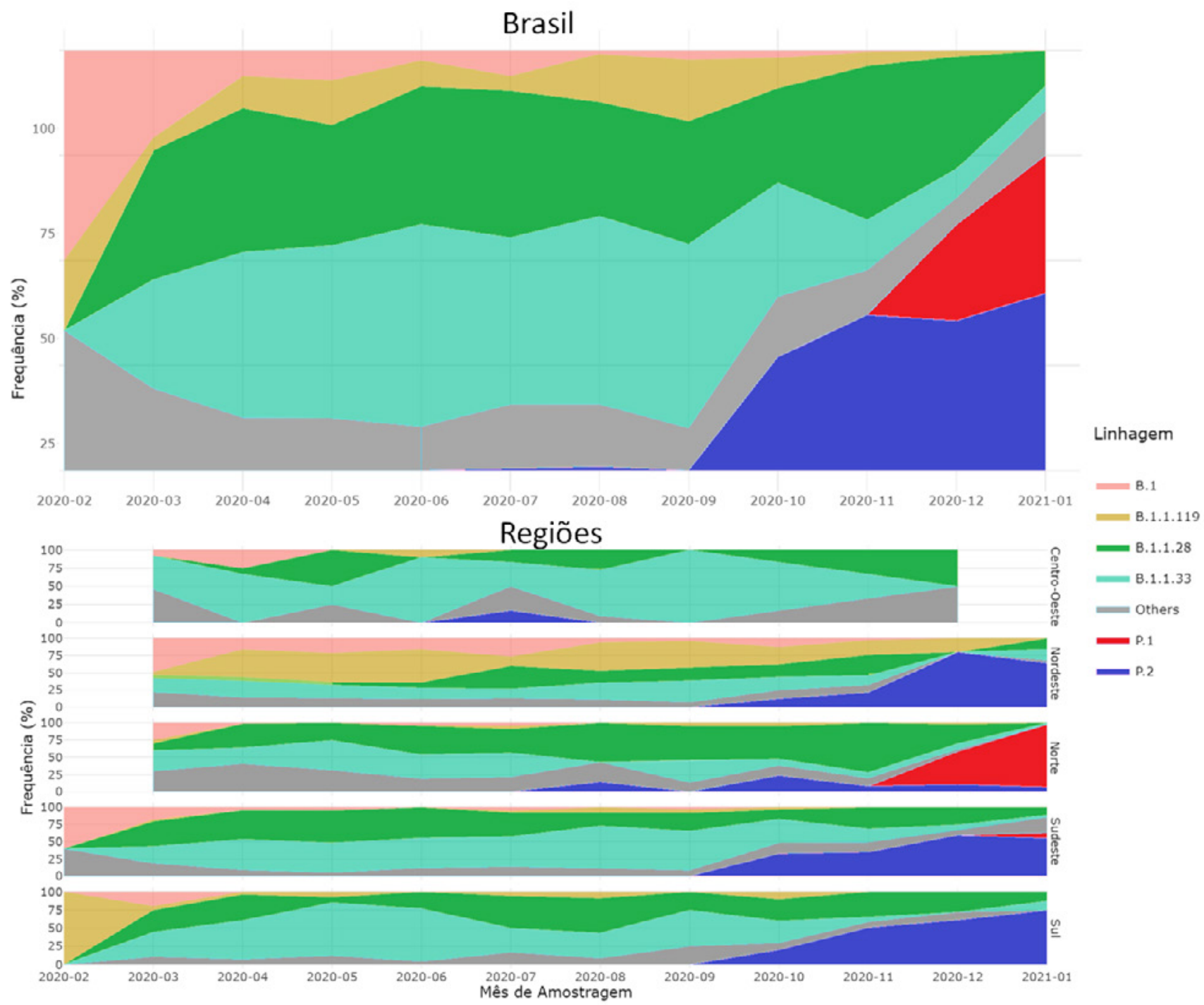

Figura 1. Distribuição percentual das principais linhagens de SARS-CoV-2 por mês de amostra. Brasil, fevereiro de 2020 a janeiro de 2021. (Fonte: Rede Genômica Fiocruz. Disponível em: https://bit.ly/2YmCSjH. Acessado em 7 de fevereiro de 2021). 
Table 2. Síntese das novas linhagens emergentes e suas características

\begin{tabular}{|c|c|c|c|c|c|c|c|c|c|}
\hline & & & \multicolumn{4}{|c|}{ Mutações } & \multicolumn{3}{|c|}{ Características clínico-epidemiológicas } \\
\hline Linhagens & $\begin{array}{l}\text { Local de infecção } \\
\text { da primeira } \\
\text { descrição }\end{array}$ & $\begin{array}{l}\text { Data da } \\
\text { descrição }\end{array}$ & N501Y & $E 484 K$ & $K 417 N$ & $K 417 T$ & $\begin{array}{l}\text { Rápida } \\
\text { expansão }\end{array}$ & $\begin{array}{l}\text { Aumento na } \\
\text { gravidade }\end{array}$ & Escape viral \\
\hline B.1.1.7 & Reino Unido & nov/20 & Sim & & & & Sim & Sim & Pouco \\
\hline B. 1.351 & África do sul & $\mathrm{dez} / 20$ & Sim & Sim & Sim & & Sim & não estudado & Sim \\
\hline P.1 & Manaus & $\mathrm{jan} / 21$ & Sim & Sim & & Sim & Sim & não estudado & $\begin{array}{l}\text { Provável (ainda } \\
\text { não estudado) }\end{array}$ \\
\hline P.2 & Rio de Janeiro & $\mathrm{dez} / 20$ & & Sim & & & Sim & não estudado & $\begin{array}{l}\text { Provável (ainda } \\
\text { não estudado) }\end{array}$ \\
\hline
\end{tabular}

